"NOTICE: this is the author's version of a work that was accepted for publication in BioChip Journal. Changes resulting from the publishing process, such as peer review, editing, corrections, structural formatting, and other quality control mechanisms may not be reflected in this document. Changes may have been made to this work since it was submitted for publication. A definitive version will be subsequently published in BioChip Journal, [Vol.7(4), pp.367-374, (2013)]."

\title{
A Microfluidic Device for Partial Cell Separation and Deformability Assessment
}

\author{
Diana Pinho ${ }^{1,2}$, Tomoko Yaginuma ${ }^{1} \&$ Rui Lima ${ }^{* 1,2}$ \\ ${ }^{1}$ Polytechnic Institute of Bragança, ESTiG/IPB, C. Sta. Apolonia, 5301-857 Bragança, Portugal. \\ ${ }^{2}$ CEFT, Faculdade de Engenharia da Universidade do Porto (FEUP), R. Dr. Roberto Frias, 4200-465 Porto, Portugal. \\ * Correspondence and requests for materials should be addressed to R. Lima (ruimec@ipb.pt)
}

\begin{abstract}
Blood flow in microcirculation shows several interesting phenomena that can be used to develop microfluidic devices for blood separation and analysis in continuous flow. In this study we present a novel continuous microfluidic device for partial extraction of red blood cells (RBCs) and subsequent measurement of RBC deformability. For this purpose, we use polydimethylsiloxane (PDMS) microchannels having different constrictions $(25 \%, 50 \%$ and $75 \%)$ to investigate their effect on the cell-free layer (CFL) thickness and separation efficiency. By using a combination of image analysis techniques we are able to automatically measure the CFL width before and after an artificial constriction. The results suggest that the CFL width increases with enhancement of the constriction and contributes to partial cell separation. The subsequent measurements of RBCs deformation index reveal that the degree of deformation depends on the constriction geometries and hematocrit after the cell separation module. The proposed microfluidic device can be easily transformed into a simple, inexpensive and convenient clinical tool able to perform both RBC separation and deformability analysis in one single device. This would eliminate the need for external sample handling and thus reducing associated labor costs and potential human errors.
\end{abstract}

Keywords: Biomicrofluidics, Microfluidic devices, Microcirculation, Blood on chips, Red blood cells, Cell separation, Cell deformability, Deformation index.

\section{Introduction}

Cell separation and identification are essential in a variety of biomedical applications including cell biology, diagnostic and therapeutic methods. Blood is a non-Newtonian fluid containing extremely rich amount of information about the physiological and pathological state of the human body. However, due to its complexity there are few accurate analysis methods. Most of the standard techniques used cell separation and sorting are often labor intensive or require additional external labels to identify cells.

Blood flow in microcirculation shows several interesting phenomena that can be used to develop microfluidic devices for blood separation and analysis in continuous flow. These phenomena include plasma skimming [1], cell-free layer (CFL) [2, 3, 4], leukocyte margination [5] and bifurcation law [6]. Recently, several researchers have used these effects and replicated in microfluidic systems. In microchannels RBCs, due to their deformability and lift forces, tend to be concentrated around the center of the microchannels while white blood cells (WBCs) and rigid RBCs (such as Malaria RBCs) tend to migrate to the CFL originated at regions close to the walls [1-5]. Bifurcation law [6] states that RBCs behavior, in microchannels with bifurcations, tends to be oriented to the wide microchannel. A number of microfluidic devices have been developed to take advantage of these natural hemodynamics phenomena. Shevkoplyas et al. [7] developed a microdevice to isolate WBCs from a blood sample by using the margination effect, whereas Hou et al. [8] have very recently proposed a biomimetic separation device to separate normal RBCs from malaria infected RBCs. Other researchers have found several advantages to control and manipulate blood flow in microfluidic devices. Fujiwara et al. [9] have found evidence that it is possible to create an artificial CFL under appropriate hemodynamic and geometrical conditions, and also the CFL thickness is strongly influenced by the RBC deformability. 
Recently, Ishikawa et al. [10] and Leble et al. [11] have shown the existence of thin CFL in the centre of the microchannel, just downstream of a confluence. This phenomenon is due to the existence of a CFL in both inner walls and a consequent formation of a triangular CFL in the region of the confluence apex [10, 11]. Faivre et al. [12] and Sollier et al. [13] have demonstrated that the CFL could be enhanced by using a microchannel containing a constriction followed by sudden expansion to separate plasma from the whole in vitro blood. However, most of these studies aim at the complete extraction of cells from plasma, which is not the case of the present study.

The main objective of the proposed microfluidic device is to separate certain amount of RBCs from plasma and then measure the deformability of individual RBCs downstream in one single step. RBC deformability is important in a clinical sense as it is related to several diseases such as diabetes, malaria, as well as cardiovascular disorders [14]. These diseases are at times fatal and early detection is crucially preferable. In this sense, $\mathrm{RBC}$ deformability can be a new biomarker and the fast and easy measurement methods are required. Former studies on RBC deformability have introduced some methodologies such as micropipette aspiration and optical tweezers where basically RBCs are stretched and the pressure or force for their extension is measured [15, 16]. Whilst these studies have revealed useful RBCs mechanical properties, these methods reqire large amount of preparation and are very time consuming. For fast analysis of vast amount of blood samples, these traditional measurement methods are unlikely to be appropriate. Moreover, since the preceded cell separation process is needed, the microfluidic devices which are easy to manipulate their microchannel geometries are more suitable for our purposes. A typical microdevice for RBC deformation studies uses a microchannel having a shape of sudden constriction in order to elongate cells. Zhao et al. [17] used a straight microchannel with a sudden narrowing and expanding constriction. Lee et al. [18], Yaginuma et al. [19-20] and Faustino et al. [21], on the other hand, used microchannels with a hyperbolic shape contraction followed by a sudden expansion region. These microfluidic experiments showed high deformability of RBCs when travelling through a contraction region. However, those studies did not include the preceded cell separation process as they have solely performed deformability measurements after an additional sample preparation [19-21].

The proposed microfluidic device aims to obtain a CFL with a low enough RBC concentration to perform cell deformability measurements downstream the separation constriction. This study is divided in two main parts. Firstly, a simple microfluidic device with different constrictions $(25 \%, 50 \%$ and $75 \%$ ) is used to test the RBCs separation. Secondly, a more complex device, able to perform in a single step both RBC separation and deformability analysis, was tested. This first tentative to integrate in one single device both tasks of separation and deformation have shown not only the viability of this new clinical strategy but also new findings that will be crucial to optimize the design for this kind of microfluidic device.

\section{Materials and methods}

\section{Working fluids}

The working fluid used in this study was dextran 40 (Dx40) containing about 9\% (i.e. Hematocrit, Hct =9) by volume of human RBCs. More details can be found in Supplementary Materials.

\section{Microdevice geometries}

The microfluidic devices tested in this study were fabricated using a soft lithography technique and consist of two main parts: a cell separation region and a cell deformation region. The microchannel height was measured by a profilometer to be $51 \mu \mathrm{m}$.

\section{Fabrication of the microfluidic devices}

The polydimethylsiloxane (PDMS) rectangular microchannels were fabricated using a soft lithographic technique. A detailed description of the fabrication process can be found elsewhere [22, 23]. Briefly, the microchannel geometry was drawn using Autocad, and a high resolution photomask was manufactured. The solid master was then fabricated on a silicon wafer with an ultrathick photoresist (SU-8 50; Kayaku MicroChem, Japan). The PDMS prepolymer was prepared by mixing a comercial prepolymer and catalyzer (Silpot 184; Dow Corning, USA) at a weight ratio of 10:1. After the mixture was degassed under vacuum, the PDMS was poured into the SU-8 photo-resist master mold and cured by baking for about $2 \mathrm{~h}$ at $70^{\circ} \mathrm{C}$. Both master and PDMS were cooled to room 
temperature and the PDMS was peeled from the master. The input/output ports are made by means of micro-pipette tips. Finally, the PDMS was washed with ethanol and brought into contact with a clean slide glass, where a reversible seal formed spontaneously.

\section{Cell separation(CS) microfluidic device}

To study the effect of a single constriction on the cell separation, a simple microfluidic device with different constriction sizes was developed. This first device had only a separation region that consists of a straight microchannel with $100 \mu \mathrm{m}$ wide $\left(w_{1}\right)$ with different constriction regions of $75 \mu \mathrm{m}, 50 \mu \mathrm{m}$ and $25 \mu \mathrm{m}$ wide $\left(w_{2}\right)$. Figure 6a shows the microfluidic device with the different contractions used to test the RBCs separation.

\section{Cell separation and deformation (CSD) microfluidic device}

To investigate the ability to perform in a single step both RBC separation and deformability analysis, a microfluidic device having a cell separation region followed by an outlet cell deformation region was tested. Note that this latter region has a concentration of cells lower than the feed Hct. This tested device had feed microchannel of $100 \mu \mathrm{m}\left(w_{1}\right)$, a constriction of $50 \%\left(w_{2}=50 \mu \mathrm{m}\right)$ followed by different kind of constrictions to perform RBC deformability. More detailed information about the dimensions can be seen in Figure 1 in supplementary materials.

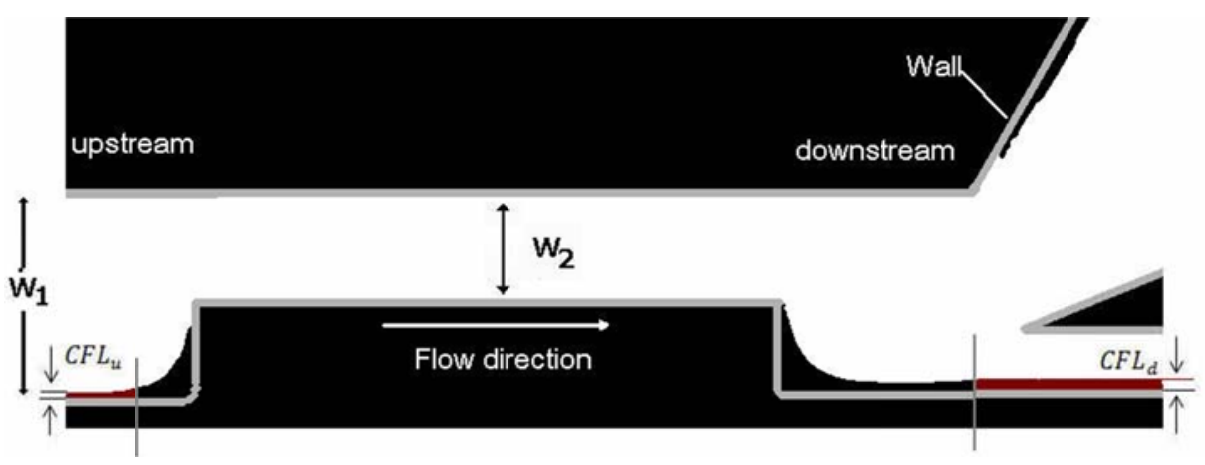

Figure 1. Schematic view of the contraction-expansion geometry, identifying the relevant variables.

\section{Experimental set-up}

In this study we have used a high-speed video microscopy system. The details of the experimental system and the image analysis procedure are explained in supplementary materials.

\section{Contraction ratio, CFL thickness and deformation index (DI)}

In this study we used microchannels with the width $\left(w_{1}\right)$ of the feed channel of $100 \mu \mathrm{m}$ and the width $\left(w_{2}\right)$ of the constritions varying from $25 \mu \mathrm{m}, 50 \mu \mathrm{m}$, up to $75 \mu \mathrm{m}$. To examine the effect of these three different artificial constrictions $(25 \%, 50 \%$ and $75 \%)$ on the CFL thickness, the contraction ratio $\left(r_{C}\right)$ was defined as follows:

$$
r_{C}=\frac{W_{2}}{W_{1}}
$$

Note that, the $r_{C}$ of the constrictions $25 \mu \mathrm{m}, 50 \mu \mathrm{m}$ and $75 \mu \mathrm{m}$ corresponds to $0.25,0.5$ and 0.75 , respectively.

To analyze the CFL thickness, measurements were taken upstream and downstream the artificial constrictions, as show in Figure 7. $\mathrm{CFL}_{\mathrm{u}}$ corresponds to the thickness of the CFL upstream the constriction, and the $\mathrm{CFL}_{\mathrm{d}}$ to the thickness downstream the constriction. The difference of the CFL upstream and downstream of the constriction was given by:

$$
\Delta_{C F L}=C F L_{d}-C F L_{u}
$$


For characterizing deformability of RBCs, we use deformation index (DI) which is defined as follows.

$$
D I=\frac{\left(L_{\text {major }}-L_{\text {minor }}\right)}{\left(L_{\text {major }}+L_{\text {minor }}\right)}
$$

Note that $L_{\text {major }}$ and $L_{\text {minor }}$ are the major and minor axis lengths of the RBC. We also calculated the length error which might be caused by the camera's exposure time but the values were sufficiently low $(\ll 1 \mu \mathrm{m})$ throughout the measured regions so that these possible deviation was ignored.

\section{Results}

\section{CS microfluidic device: constriction effect in the CFL thickness}

In the CS device we have evaluated the effect of the constriction on the CFL thickness and consequently on the separation effectiveness. This study was performed with different $r_{C}(0.25,0.5$ and 0.75$)$, at a Hct of $9 \%$ and with three different flow rates $(1 \mu \mathrm{l} / \mathrm{min}, 5 \mu \mathrm{l} / \mathrm{min}$ and $10 \mu \mathrm{l} / \mathrm{min})$.

Qualitative flow visualization results show that all constrictions enhance CFL thickness. Furthermore, it is clear that the enhancement is more pronounced for the microchannel with $\mathrm{r}_{\mathrm{C}}=0.25$. To obtain more detail results about the constriction effect, quantitative measurements of the CFL thickness were taken both upstream $\left(\mathrm{CFL}_{\mathrm{u}}\right)$ and downstream $\left(\mathrm{CFL}_{\mathrm{d}}\right)$ the constrictions at different flow rates (see Figure 2).

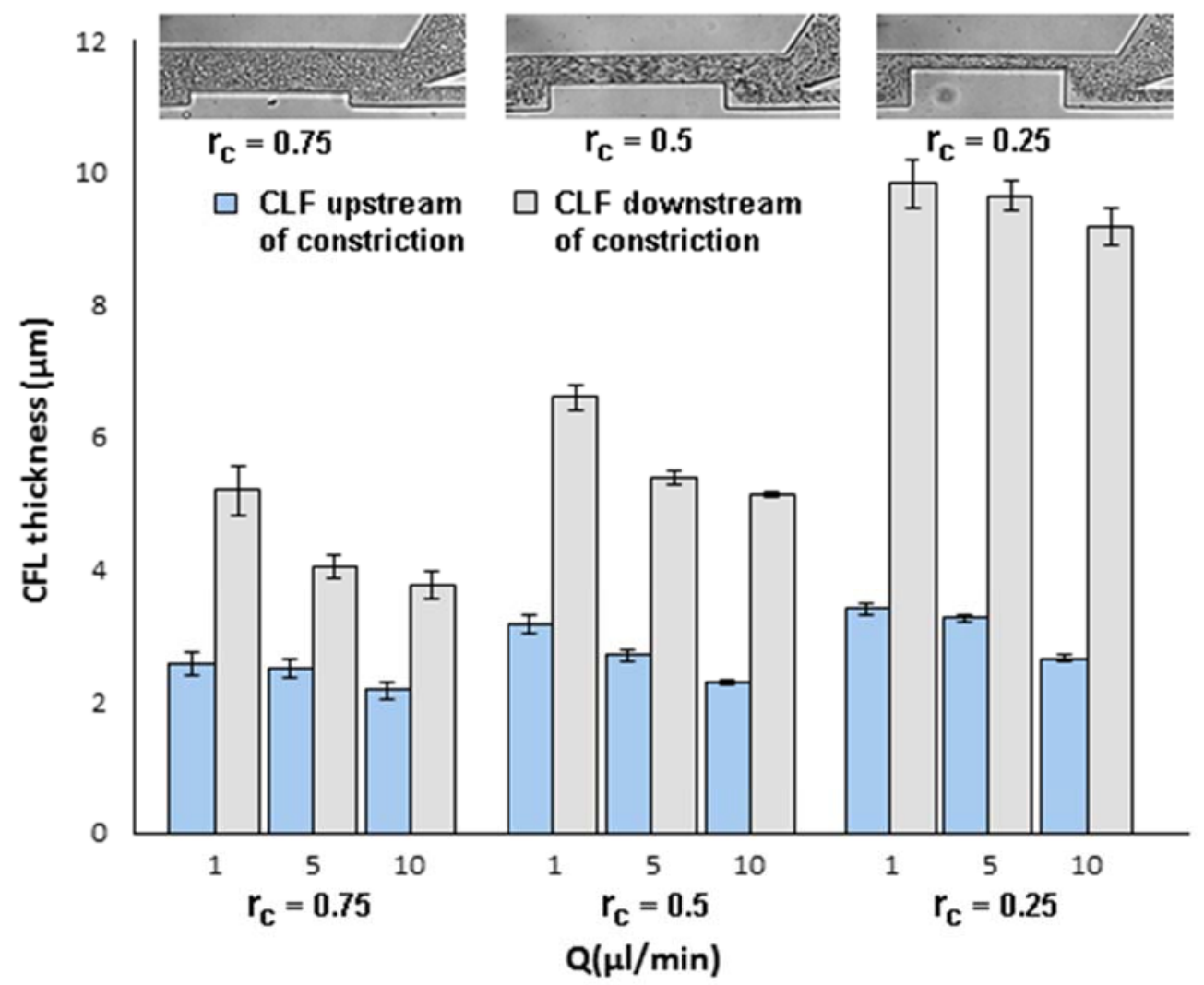

Figure 2. CFL thickness for both upstream $\left(\mathrm{CFL}_{\mathrm{u}}\right)$ and downstream $\left(\mathrm{CFL}_{\mathrm{d}}\right)$ constrictions at different flow rates $(1 \mu \mathrm{l} / \mathrm{min}, 5 \mu \mathrm{l} / \mathrm{min}$ and $10 \mu \mathrm{l} / \mathrm{min})$ and different constriction ratios $\left(\mathrm{r}_{\mathbf{C}}=0.25, \mathrm{r}_{\mathrm{C}}=0.5\right.$ and $\left.\mathrm{r}_{\mathrm{C}}=0.75\right)$.

Overall the quantitative measurements of the CFL thickness, presented in Figure 2, show clearly that all the constrictions tested enhance the CFL thickness at downstream region $\left(\mathrm{CFL}_{\mathrm{d}}\right)$. It is also clear that the $\mathrm{CFL}_{d}$ increases with decreasing $\mathrm{r}_{\mathrm{C}}$ and this enhancement is more pronounced for $\mathrm{r}_{\mathrm{C}}=$ 0.25 where $\mathrm{CFL}_{\mathrm{d}}$ is about three orders of magnitude greater than the $\mathrm{CFL}_{\mathrm{u}}$. Additionally, these results show a tendency of the CFL thickness to decrease with increasing flow rate, which corroborates the results obtained using a microchannel with similar constrictions and a Hct of 2.6\% [12]. Note that, this tendency is more pronounced for $\mathrm{r}_{\mathrm{C}}=0.5$ and 0.75 . 


\section{CSD microfluidic device: constriction effect and deformation measurements}

Downstream the CS region, RBC deformation measurement region is followed in the same microchannel as shown in Figure 1 in supplementary materials. In this study, two geometrically different constriction regions, one with a sudden contraction and another with a smooth contraction, were tested in order to examine efficient deformability measurements. For this examination, the upstream constriction (CS region) with $r_{C}=0.5$, an inlet Hct of $9 \%$, and a flow rate of $1 \mu \mathrm{l} / \mathrm{min}$ was used.

The Hct at this region should be around $2.4 \%$ and RBCs tend to flow in line and the majority of them start becoming like a parachute or umbrella shape when they pass through a sudden constriction microchannel with a width of $7 \mu \mathrm{m}$. This RBC deformation behaviour is mainly due to the constriction to be smaller of the normal size of human RBC $(8 \mu \mathrm{m})$ and to the shear flow. Figure 3a shows the $\mathrm{x}$ axial DI values of one individual RBC flowing through the sudden constriction microchannel. At the contraction entrance $(\mathrm{x} / \mathrm{Lc}=0)$, the highest $\mathrm{DI}$ was obtained due to a strong shear stress caused by the sudden narrowing of the flow field. Then the RBC starts changing their shape from an ellipsoid to a parachute, reaching a low DI value at the exit of the contraction $(x / L c=1$, see also the image IV in Figure 3a.) The sudden contraction is effective to observe a strong deformation of RBCs with high shear stress. However, proceeded DI values are slightly unreliable as parachute shapes are rather variable and the definition of major and minor axis of the cell in this shape does not have the same meaning for the ellipsoid shape. Besides this, overlaps of the cells were often observed, which is not ideal for deformation measurements especially when an automatic method is applied. The Hct control has to be carefully done in the upper stream.

Figure $3 \mathrm{~b}$ shows the DI values of one single RBC flowing through the smooth contraction region for deformability measurement. It is clear that the highest DI is observed around $\mathrm{x} / \mathrm{Lc}=0.5$. In this type of constriction, in the middle of the microchannel $(\mathrm{y}=0$ and $\mathrm{z}=0)$ extensional force is likely to be more dominant over shear force, where RBCs tend to flow stably with no rotation or orientation. For good image analysis results it is important to measure RBCs flowing in the same orientation so that this condition is ideal.
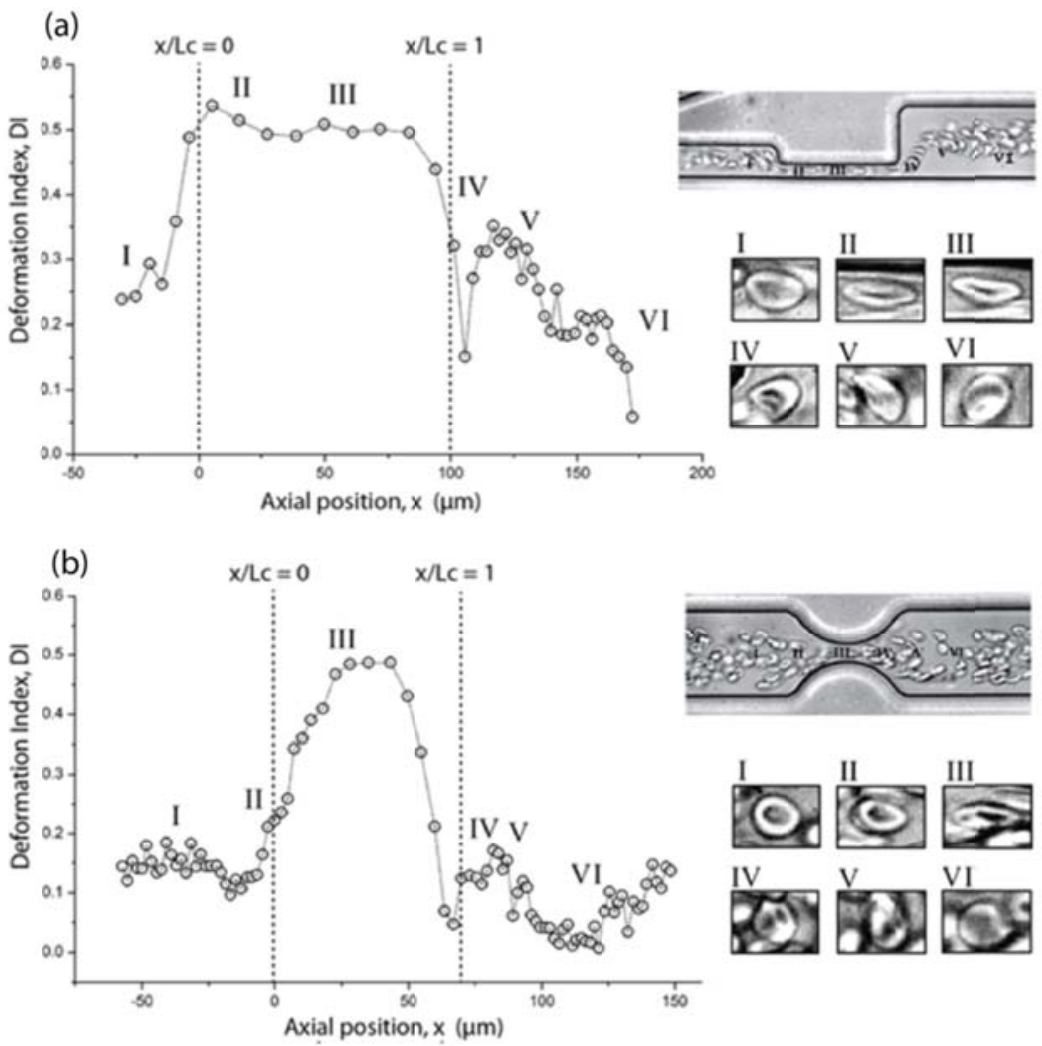

Figure 3. DI values of a single RBC flowing through (a) a sudden contraction and (b) a smooth contraction. 


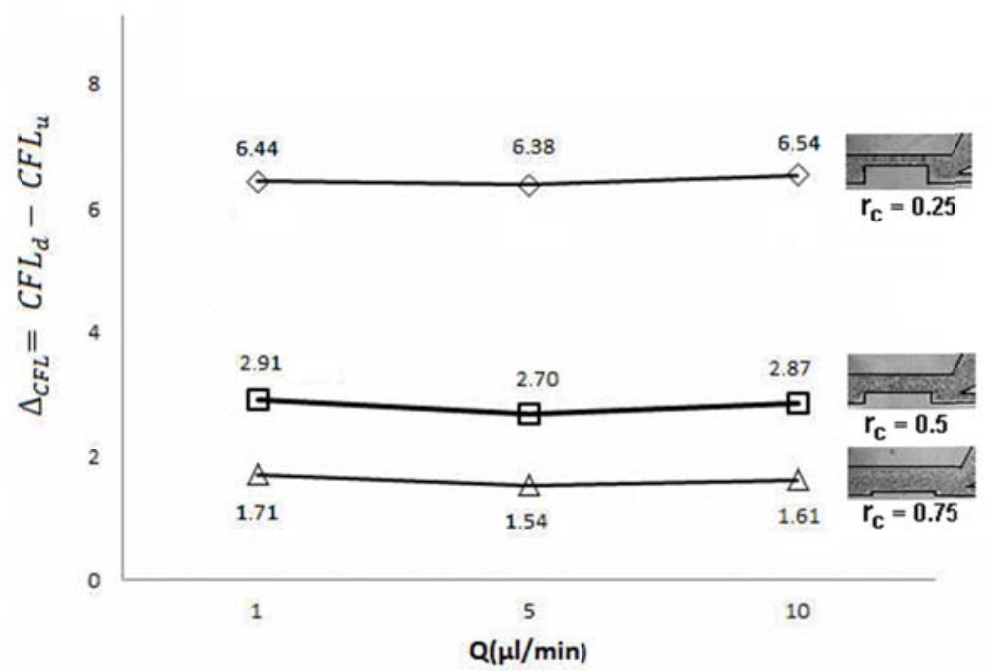

Figure 4. The difference of the CFL upstream and downstream of the constriction $\left(\Delta_{C F L}\right)$ at different flow rates and different constriction ratios $\left(r_{C}=0.25, r_{C} 0.5\right.$ and $\left.r_{C} 0.75\right)$.

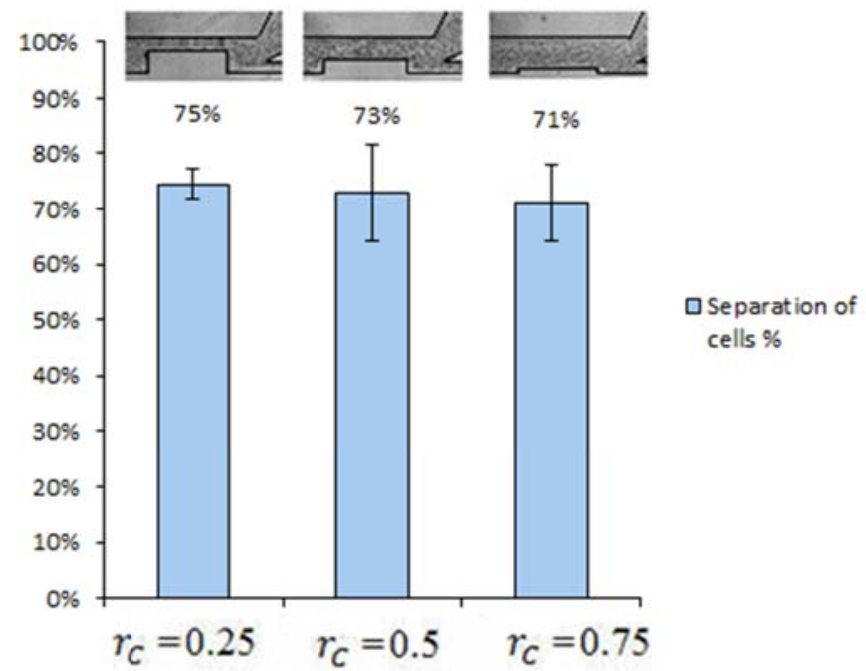

Figure 5. Separation rate of the RBCs flowing to the outlet region number 2 (upper side outlet). The measured values are expressed as the mean \pm standard deviation according to a t-test analysis at a $95 \%$ confidence interval.

\section{Discussion}

To analyse in more detail the effect of the artificial constrictions on the CFL thickness, Figure 4 shows the $\Delta_{C F L}$ for each constriction as a function of the flow rate. These results show clearly that $\Delta_{C F L}$ increases with decreasing the $r_{C}$ and this enhancement is more pronounced for $r_{C}=0.25$ where $\Delta_{C F L}$ is about two to four orders of magnitude greater than $\Delta_{C F L}$ for $r_{C}=0.5$ and $r_{C}=0.75$, respectively. It is worth mentioning that the highest $\Delta_{C F L}$ corresponds to $\mathrm{r}_{\mathrm{C}}=0.25$ and is independent of the flow rate. For $r_{C}=0.5$ and $r_{C}=0.25$ for low flow rate there is a tendency to decrease but tend to flattened to higher flow rates. However these results show evidence that the constriction has a strong impact in the CFL thickness (see Figure 5).

Figure 5 shows the separation rate of cells flowing to the outlet region number 2 (see Figure 6), i. e., for $r_{C}=0.5$ this constriction can efficiently separate about $73 \%$ of blood cells to region 2 and remaining $27 \%$ of cells to the deformation region. This separation estimation is based on the same image analysis approach used to calculate the CFL thickness. Briefly, we have used binary images and considered that all the white part, upstream the constriction, corresponds to a uniform distribution of 
the RBCs core to be $100 \%$. Hence, by measuring the CFL thickness downstream the constriction we were able to estimate the separation rate depending on the size of the constriction. As expected, the constriction with higher efficiency was the one with $r_{C}=0.25$. Although statistical analysis have shown a significant difference between $r_{C}=0.25$ and $r_{C}=0.75$, this is not the case for $r_{C}=0.5$. Hence, we believe that the most effective way to get a considerable difference between $r_{C}=0.25$ and $\mathrm{r}_{\mathrm{C}}=0.5$ is by decreasing the geometrical parameter $\mathrm{L}_{2}$ (see Figure 6 ). In the present study we have decided to use the constriction with $\mathrm{r}_{\mathrm{C}}=0.5$ to test the ability of the proposed device to perform in one single step both separation and deformation of blood cells. Note that for $\mathrm{r}_{\mathrm{C}}=0.5$ and by considering a feed Hct to be about $9 \%$, the local Hct in the outlet 3 (deformation region) is around $2.4 \%$. Very recently, by using $1 \%$ Hct we have successfully measured the RBC deformability in hyperbolic contractions [20]. In order to achieve $1 \%$ Hct with the proposed microfluidic device the separation rate should be around $90 \%$ and consequently $\mathrm{r}_{\mathrm{C}}$ must be less than 0.25 . Alternatively, by decreasing the geometrical parameter $\mathrm{L}_{2}$, to a dimension less than $100 \mu \mathrm{m}$, it will be possible to achieve easily local Hcts less than $1 \%$.

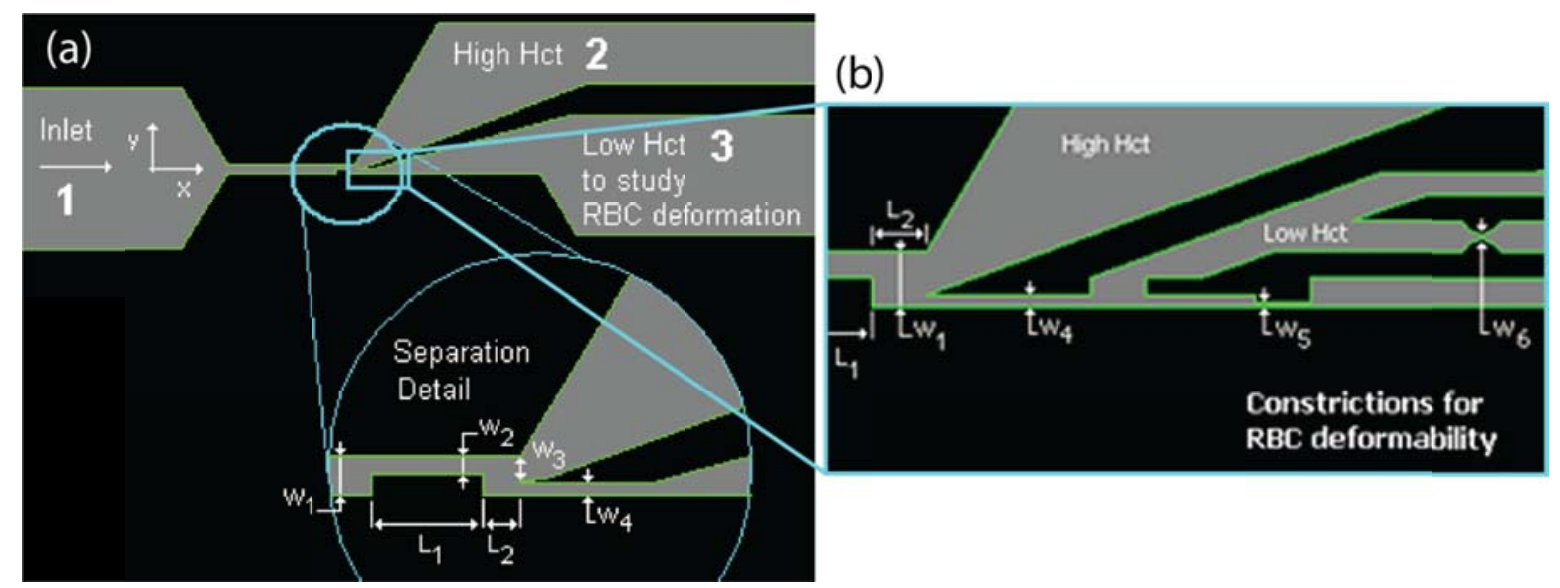

Figure 6. Schematic drawing of the microfluidic device. (a) the entire view and close-up view of cell separation part. The dimensions of $\mathrm{L}_{1}, \mathrm{~L}_{2}, \mathrm{w}_{1}, \mathrm{w}_{3}, \mathrm{w}_{4}$, are $300 \mu \mathrm{m}, 100 \mu \mathrm{m}, 100 \mu \mathrm{m}, 80 \mu \mathrm{m}$ and 20 $\mu \mathrm{m}$, respectively. The different constrictions were used ( $w_{2}=75 \mu \mathrm{m}, 50 \mu \mathrm{m}$ and $\left.25 \mu \mathrm{m}\right)$. (b) the view of deformation measurement part. The dimensions of $w_{5}$ and $w_{6}$ are $7 \mu \mathrm{m}$ and $10 \mu \mathrm{m}$, respectively. The height of the microchannel is $51 \mu \mathrm{m}$.
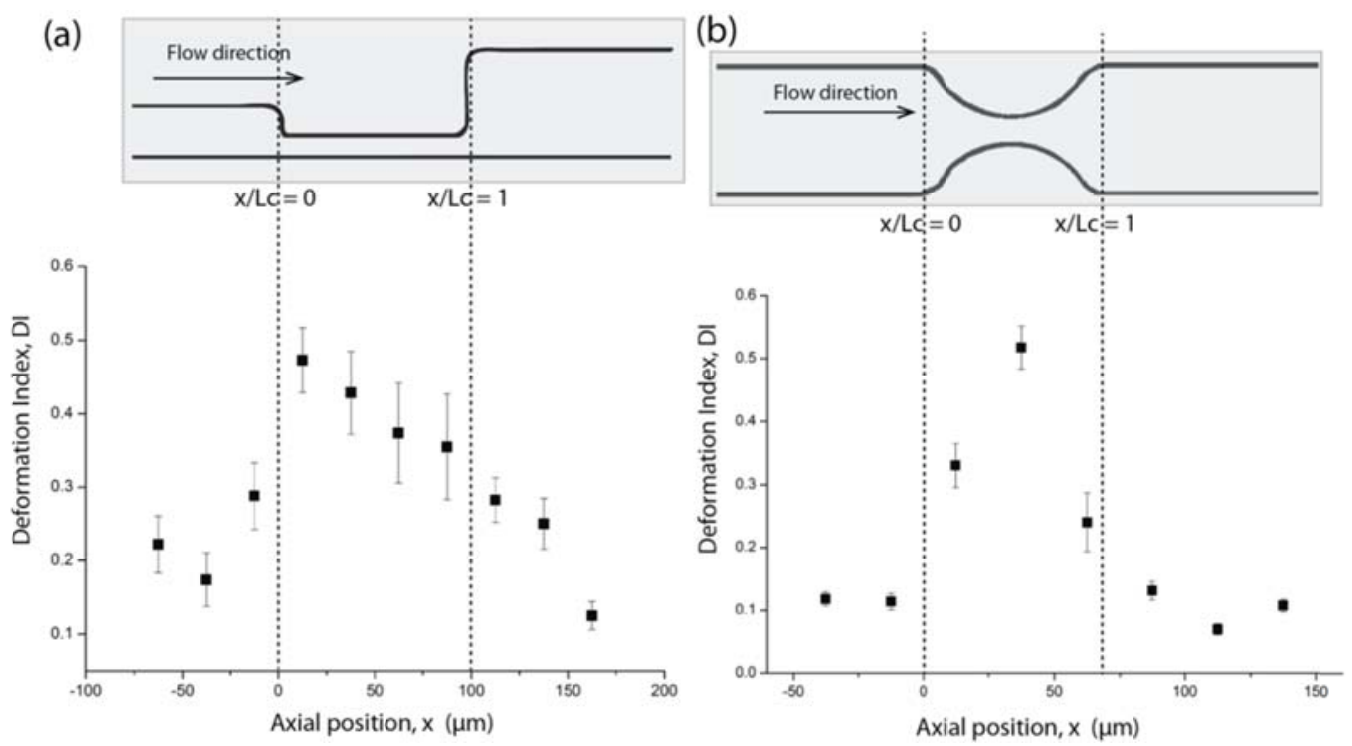

Figure 7. Mean DI values of two deformation measurement modules: (a) a sudden and (b) a smooth contractions. 
In terms of the deformation measurements, Figure 7 shows average DI values of ten RBCs flowing through the two different constriction regions. The maximum DI value is in both cases relatively the same, however, for the sudden contraction case (a) the maximum DI is located close to the constriction entrance whereas for the smooth case (b) the maximum DI is located around the middle of the constriction. In addition, in the constricted region in (a) RBCs tend to flow as parachute like shape and the DI values are not truly comparative in the sequence of cell behaviour throughout the deformation analysis region of the device. At least, the region for measurements has to be carefully selected for a meaningful comparison. On the other hand, the smooth contraction provides less deviation of DI results and indicates clearly an appropriate measurement region $(\mathrm{x} / \mathrm{Lc}=0.5)$, i. e., middle of constriction. The majority of the cells are flowing with the same orientation at the centreline of the channel where the cells are under an extensional flow dominated regime and as a result they tend to elongate in y direction induced mainly by this extensional force. This stable state of the RBCs makes this geometry suitable to measure small changes of RBC deformability. More detailed studies on RBCs extensional flow effects can be found elsewhere [19-21].

As mentioned before, by using a constriction with $r_{C}=0.5$, we have observed collisions and the overlap of neighbouring cells flowing within the measurement region. In the CS region, the Hct of the flowing fluid is reduced from $9 \%$ to $\approx 2.4 \%$ but ideally it needs to be less than that. Nevertheless, it is worth mentioning that by reducing Hct to less than $2 \%$ the number of RBCs to measure may not be large enough to obtain a significant statistical picture of the results. Therefore, an optimization of channel, i.e. change in depth, needs to be done in order to obtain the best representative results of the RBCs DI.

\section{Conclusions}

The conventional microfluidic methods for measuring RBC deformability are often labor intensive and require additional sample modification and preparation. In this paper, we present a new, simple microfluidic device able to perform both RBCs separation and deformability assessment in one single step. In general, our results indicate that the proposed device can perform both operations (separation and deformation) successfully. The reported results show evidence that the constriction has a strong impact on the CFL thickness and consequently on separation rate. Moreover, the deformability results show clearly that most appropriate geometry to measure RBC deformability is the microchannel containing a smooth constriction region. In this kind of geometry due to the existence of a dominant extensional force the majority of the RBCs tend to flow with the same orientation. This stable performance of the RBCs may prove to be enough sensitive to detect small changes of RBC deformability and thus it may have the ability to diagnose early stage RBC related diseases such as diabetes, malaria and sickle cell anemia. Therefore, the integrated and simple continuous system operations make the proposed microfluidic device a potential diagnostic technique to be applied to both healthy cells and blood cell diseases.

\section{Acknowledgement}

The authors acknowledge the financial support provided by 2007 Global COE Program "Global NanoBiomedical Engineering Education and Research Network", Japan and grant-in-Aid for Science and Technology PTDC/SAU-BEB/108728/2008, PTDC/SAU-BEB/105650/2008, PTDC/EME-MFE/099109/2008, PTDC/SAUENB/116929/2010 and scholarship SFRH/BD/89077/2012 from FCT (Science and Technology Foundation), COMPETE, QREN and European Union (FEDER). The authors are also very grateful to Dr. Mónica Oliveira (Strathclyde University), Professor Takuji Ishikawa and Professor Takami Yamaguchi (Tohoku University) for their suggestions and support to this research work.

\section{References}

[1] Caro, C., Pedley, T., Schroter, R. \& Seed, W. The Mechanics of the Circulation. Oxford University Press, 1978.

[2] Lima, R. et al. Measurement of individual red blood cell motions under high hematocrit conditions using a confocal micro-PTV system. Ann Biomed Eng. 37, 1546-59, (2009).

[3] Fujiwara, H., et al. Red blood cell motions in high-hematocrit blood flowing through a stenosed microchannel. J Biomech 42, 838-843, (2009). 
[4] Lima, R., et al. Axisymmetric polydimethysiloxane microchannels for in vitro hemodynamic studies. Biofabrication, 1: 1-7, (2009).

[5] Goldsmith, H., \& Spain, S. Margination of leukocytes in blood flow through small tubes. Microvasc Res, 27, 2, 204-222, (1984).

[6] Yang, S., Undar, A., \& Zahn, J.D. A microfluidic device for continuous, real time blood plasma separation. Lab Chip 6:871, (2006).

[7] Shevkoplyas, S.S., Yoshida, T., Munn, L.L., \& Bitensky, M.W. Biomimetic Autoseparation of Leukocytes from Whole Blood in a Microfluidic Device. Anal Chem, 77, 933-937, (2005).

[8] Hou, H.W., et al. Deformability based cell margination - A simple microfluidic design for malaria-infected erythrocyte separation. Lab Chip, 10, 2605-2613, (2010).

[9] Fujiwara, H., et al. Red blood cell motions in high-hematocrit blood flowing through a stenosed microchannel. J Biomech 42, 838-843, (2009).

[10] Ishikawa, T., et al. Asymmetry of blood flow and cancer cell adhesion in a microchannel with symmetric bifurcation and confluence. Biomed Microdevices, 13, 159, (2011).

[11] Leble, V., et al. Asymmetry of red blood cell motions in a microchannel with a diverging and converging bifurcation. Biomicrofluidics, 5, 044120, (2011).

[12] Faivre, M., Abkarian, M., Bickraj, K. \& Stone, H. Geometrical focusing of cells in a microfluidic device: an approach to separate blood plasma. Biorheology, 43, 147-159, (2006).

[13] Sollier, E., Cubizolles, M., Fouillet, Y. \& Achard J. Fast and continuous plasma extraction from whole human blood based on expanding cell-free layer devices. Biomed Microdevices, 12, 485-97, (2010).

[14] Mokken, F.C., Kedaria, M., Henny, C.P., Hardeman, M.R., \& Gelb, A.W. The clinical importance of erythtrocyte deformability, a hemorrheological parameter. Ann Hematol, 64, 113-122, (1992).

[15] Musielak, M. Red blood cell deformability measurement: Review of techniques. Clin Hemorheol Micro, 42, 47-64, (2009).

[16] Shiga, T., Maeda, N., \& Kon, K. Erythrocyte rheology. Crit Rev Oncol Hemat, 10: 9-48, (1990).

[17] Zhao, R., et al. Microscopic investigation of erythrocyte deformation dynamics. Biorheology, 43(6), 747$765,(2006)$

[18] Lee, S.S., Yim, Y., Ahn, K.H., \& Lee, S.J. Extensional flow-based assessment of red blood cell deformability using hyperbolic converging microchannel. Biomed Microdevices, 11, 1021-1027, (2009).

[19] Yaginuma, T., Oliveira, M.S.N., Lima, R., Ishikawa, T., \& Yamaguchi, T. Red blood cell deformation in flows through a PDMS hyperbolic microchannel, Proceedings of NSTI-Nanotech, 2 505-507, (2011).

[20]Yaginuma, T., Oliveira, M.S.N., Lima, R., Ishikawa, T., \& Yamaguchi, T. Human red blood cell behavior under homogeneous extensional flow in a hyperbolic-shaped microchannel. Biomicrofluidics, 7:054110, (2013).

[21] Faustino V., Pinho D., Yaginuma T., R. Calhelha, M. Oliveira, I. Ferreira, Lima R., Flow of red blood cells suspensions through hyperbolic microcontractions. Visualization and simulation of complex flows in biomedical engineering. In: R.Lima, T. Ishikawa, Y. Imai \& M. S. N. Oliveira (Eds), Springer, 2013 (in press).

[22] Lima, R., Wada, S., Tanaka, S., Takeda, M., Ishikawa, T., Tsubota, K., Imai, Y., Yamaguchi, T., In vitro blood flow in a rectangular PDMS microchannel: experimental observations using a confocal micro-PIV system. Biomedical Microdevices, 10: 153-167, 2008.

[23] Lima, R., et al. Microscale flow dynamics of red blood cells in microchannels: an experimental and numerical analysis. In: Tavares and Jorge (Eds) Computational Vision and Medical Image Processing: Recent Trends, Springer 19, 297-309, (2011).

[24]Abramoff, M., Magelhaes, P. \& Ram, S. Image Processing with ImageJ. Biophotonics International, 7, 11, 36-42, (2004). 


\section{Supplementary Materials}

\section{MATERIALS AND METHODS}

\subsection{Working fluids}

The working fluid used in this study was dextran 40 (Dx40) containing about 9\% (i.e. Hematocrit $(\mathrm{Hct}=9 \%)$. Briefly, blood was collected from a healthy adult volunteer and heparin was added in order to prevent coagulation. The red blood cells (RBCs) were separated from bulk blood by centrifugation and aspiration of the plasma and buffy coat. The RBCs were then washed twice with a physiological saline solution and diluted with Dx40 to make up the required RBC concentration. All blood samples were stored hermetically at $4^{\circ} \mathrm{C}$ until the experiments were performed at controlled temperature of approximately $37^{\circ} \mathrm{C}$.

\subsection{Fabrication of the microfluidic devices}

The polydimethylsiloxane (PDMS) rectangular microchannels were fabricated using a soft lithographic technique. A detailed description of the fabrication process can be found elsewhere [22, 23]. Briefly, the microchannel geometry was drawn using Autocad, and a high resolution photomask was manufactured. The solid master was then fabricated on a silicon wafer with an ultrathick photoresist (SU-8 50; Kayaku MicroChem, Japan). The PDMS prepolymer was prepared by mixing a comercial prepolymer and catalyzer (Silpot 184; Dow Corning, USA) at a weight ratio of 10:1. After the mixture was degassed under vacuum, the PDMS was poured into the SU-8 photo-resist master mold and cured by baking for about $2 \mathrm{~h}$ at $70^{\circ} \mathrm{C}$. Both master and PDMS were cooled to room temperature and the PDMS was peeled from the master. The input/output ports are made by means of micro-pipette tips. Finally, the PDMS was washed with ethanol and brought into contact with a clean slide glass, where a reversible seal formed spontaneously.

\subsection{Experimental set-up}

The high-speed video microscopy system used in this study is shown in Figure 1. This system consisted mainly by an inverted microscope (IX71; Olympus, Japan) combined with a high-speed camera (Phantom v7.1; Vision Research, USA). All the microfluidic devices were placed on the stage of the inverted microscope and by using a syringe pump (KD Scientific, USA), a constant pressuredriven was maintained. Additionally, a thermo plate controller (Tokai Hit) was set to $37^{\circ} \mathrm{C}$ in order to have an environment close to in vivo conditions. 


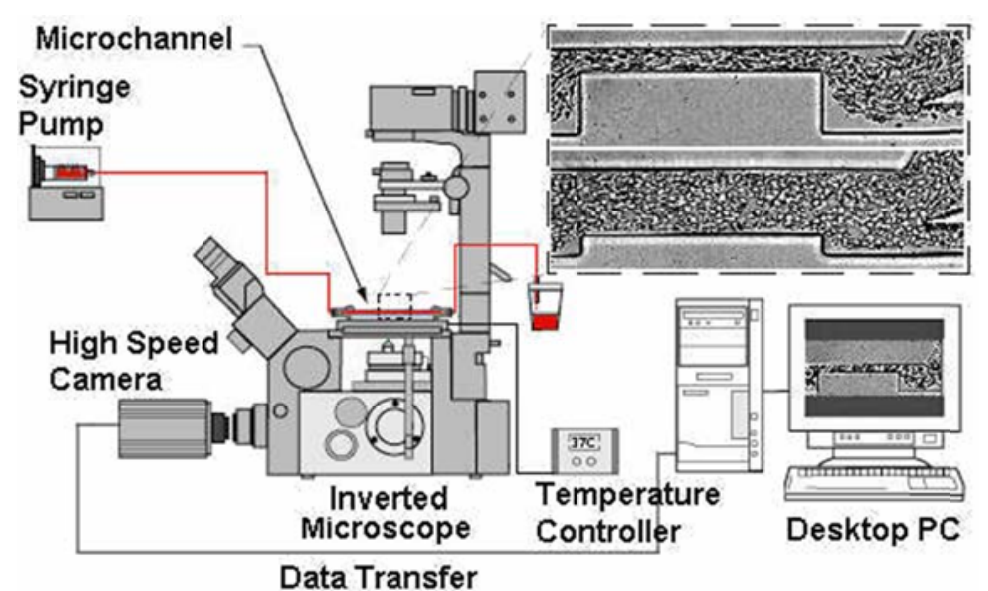

Figure 1. High-speed video microscopy system used to test the performance of the microfluidic devices.

\subsection{Image analysis}

\section{Cell separation (CS) microfluidic device}

All images were captured around the middle plane of the microchannels with a resolution of $800 \times 304$ pixels, at a rate of 8000 frames/s and an exposure time of $0.125 \mathrm{~ms}$. Figure 2 shows an original image with the flow of RBCs (halogen illumination) through a $25 \%$ constriction for a constant flow rate of 1 $\mu \mathrm{L} / \mathrm{min}$. The recorded images were transferred to the computer and then evaluated in Image $\mathrm{J}(\mathrm{NIH})$ [24].

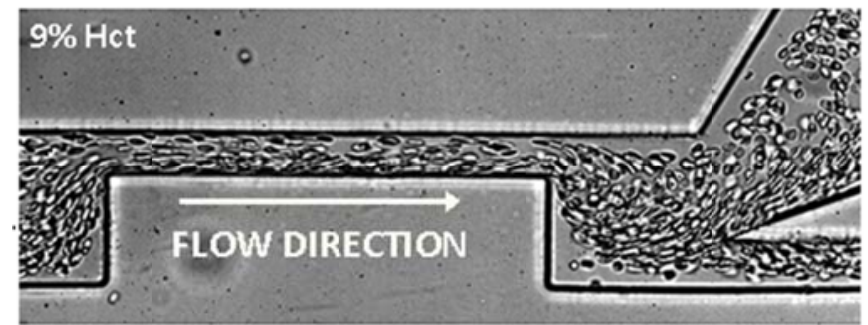

Figure 2. Original image with the flow of RBCs through a 75\% constriction for a constant flow rate of $1 \mu \mathrm{L} / \mathrm{min}$.

First, the captured videos were converted to a sequence of static images (stack). Then, each pixel's with maximum intensity of all the images in the stack was selected by using Image " $Z$ project" function, which results in a single image having a region brighter than the background. This brighter region corresponds to RBCs core. To obtain quantitative measurements the grey scale images were converted to binary images with thresholding (see Figure 3). 


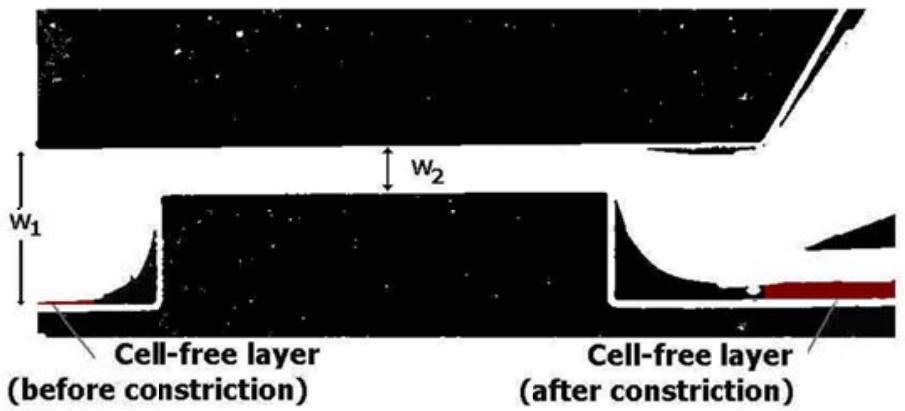

Figure 3. Binary image obtained after image processing and binarization using "Image J" software. The channel dimensions are: $w_{1}=100 \mu \mathrm{m}$ and $w_{2}=25 \mu \mathrm{m}$.

\section{Cell separation and deformation (CSD) microfluidic device}

$\mathrm{RBC}$ deformation analysis has been achieved by measuring RBC deformation index (DI) at the contraction region of the microchannel $\left(\mathrm{W}_{5}\right.$ and $\mathrm{W}_{6}$ in Figure 4), including upstream and downstream the region. Ten cells were selected for each contractions, and major and minor axis lengths of the flowing cells were measured manually frame by frame.

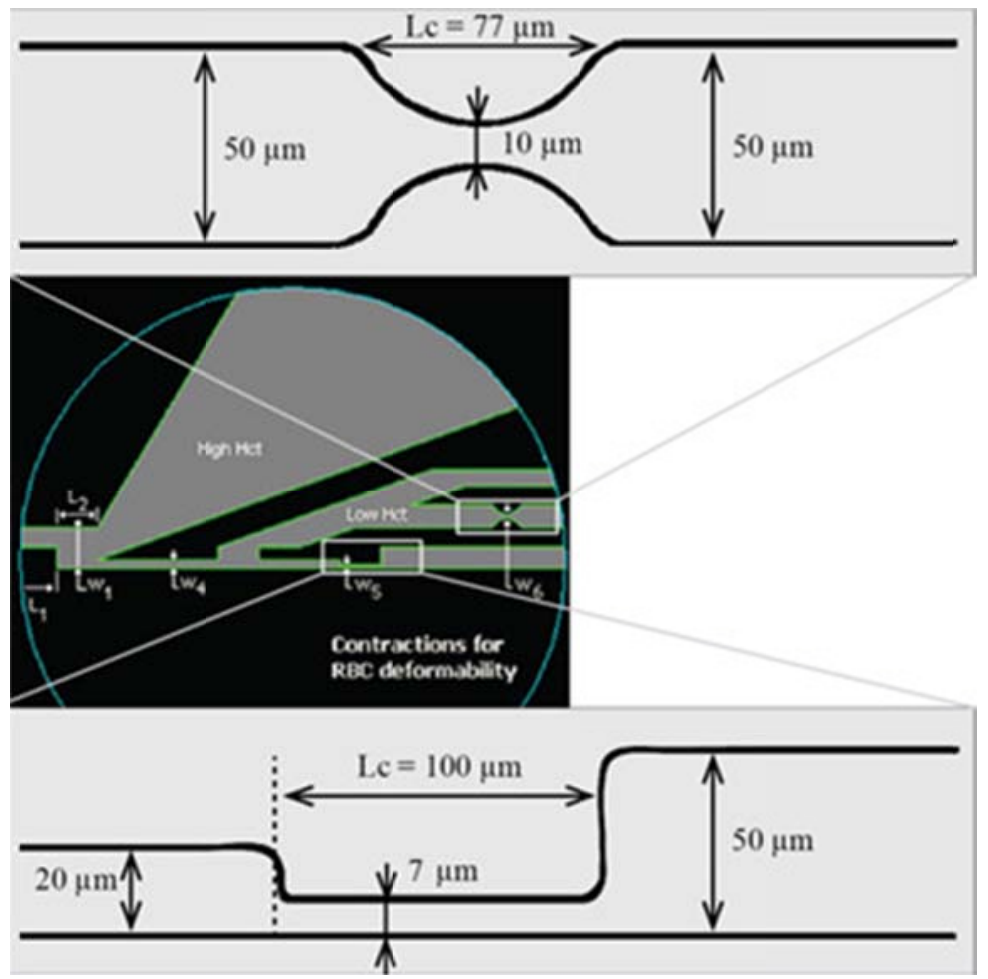

Figure 4. Schematic diagram of RBC deformation measurement regions with detailed dimensions.

\section{Deformation Index}

Deformation Index (DI) is defined as:

$$
D I=\frac{\left(L_{\text {major }}-L_{\text {minor }}\right)}{\left(L_{\text {major }}+L_{\text {minor }}\right)}
$$

where $L_{\text {major }}$ and $L_{\text {minor }}$ are the major and minor axis lengths of the RBC (see Figure 5a). For parachutelike shape RBCs, we measured the major and minor axis lengths as shown in Figure $5 b$. 
(a)

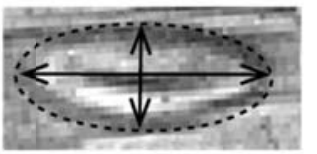

(b)

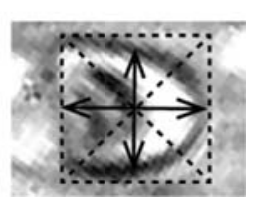

Figure 5. Schematic view of DI measurements. (a) the measurement of ellipsoid shape cells. (b) the measurement of parachute shape cells. The box surrounding the cell defines the center of the cell and the major and minor axes are defined.

The measurements were done manually with the assistance of an image handling software, ImageJ (NIH) [24]. We also calculated the length error which might be caused by the camera's exposure time. The 2D image contains the flowing cell's shadow which is captured when the camera's shutter is opened and this shadow should not be considered as the cell's actual length especially in the axis of flowing direction (y). To avoid this error the cell's traveling length (shadow) in y-axis was calculated by the RBC's actual velocity times the exposure time of the high speed camera. In order to obtain the RBC's actual velocity, we measured the traveling length of the cells frame by frame and then divided it by the frame interval time. The cell's traveling length values obtained by these calculations were found to be sufficiently low $(\ll 1 \mu \mathrm{m})$ throughout the measured regions so that these possible deviations were ignored.

\section{RESULTS}

Figure 6 shows representative original images and correspondent binary images from in vitro blood measurements obtained at different artificial constrictions $\left(\mathrm{r}_{\mathrm{C}}=0.25, \mathrm{r}_{\mathrm{C}}=0.5\right.$ and $\left.\mathrm{r}_{\mathrm{C}}=0.75\right)$.
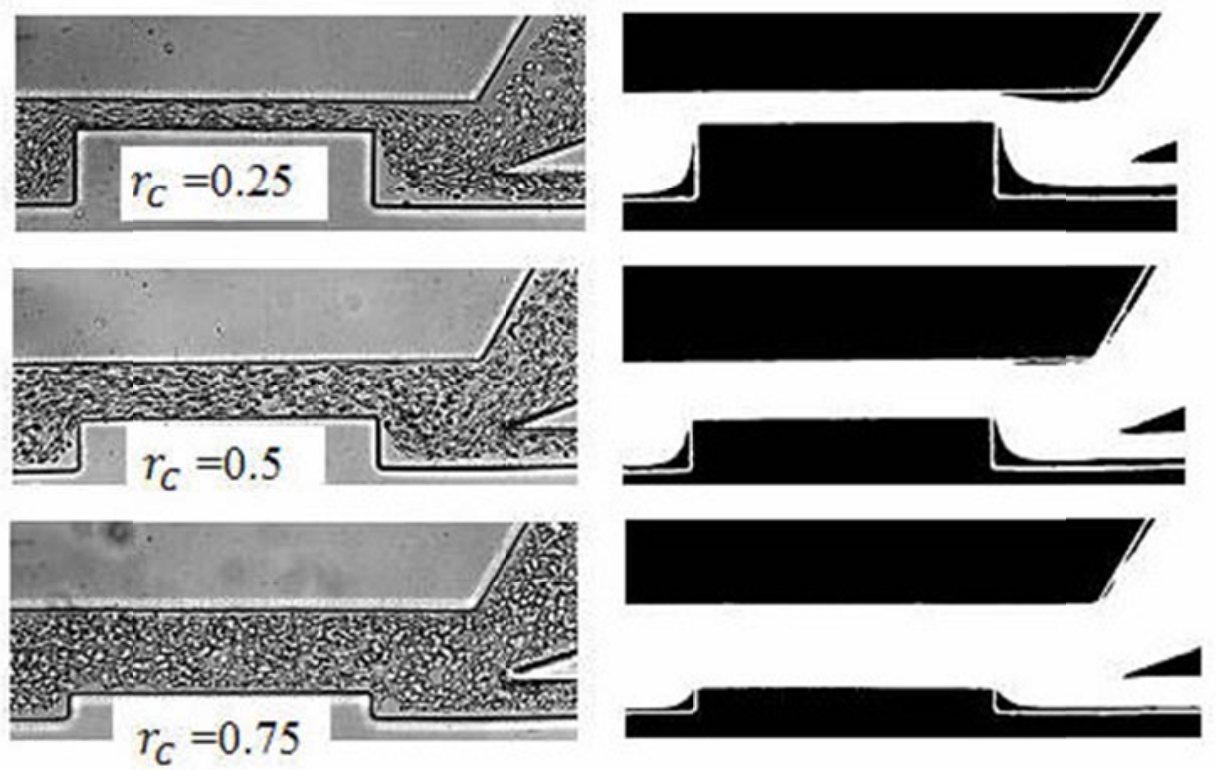

Figure 6. Original blood flow visualization images (left side) and correspondent binary images (right side) for different artificial constrictions $\left(r_{C}=0.25, r_{C}=0.5\right.$ and $\left.r_{C}=0.75\right)$.

Figure 7 shows a representative original image of the deformation region of the CSD microfluidic device upstream the separation region with $\mathrm{r}_{\mathrm{C}}=0.5$. 


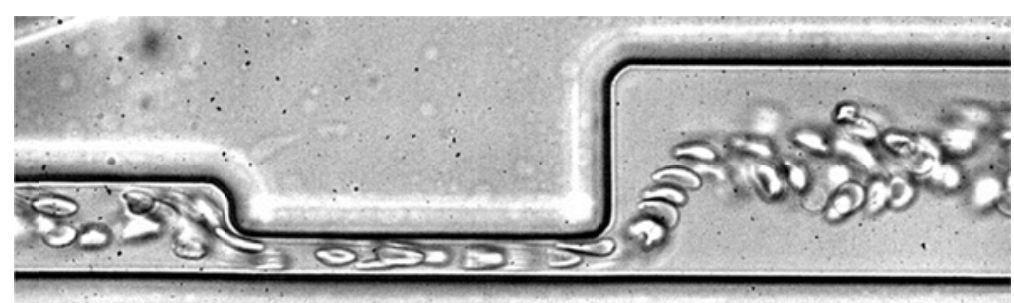

Figure 7. Blood flow visualization images of the deformation region of the CSD microfluidic device containing a sudden constriction microchannel with a width of $7 \mu \mathrm{m}$.

Figure 8 shows a representative original image of the deformation region with a smooth contraction. The Hct at this region should be also around $2.4 \%$. In this contraction, the maximum deformation of the cell can be observed at the narrowest part of the contraction.

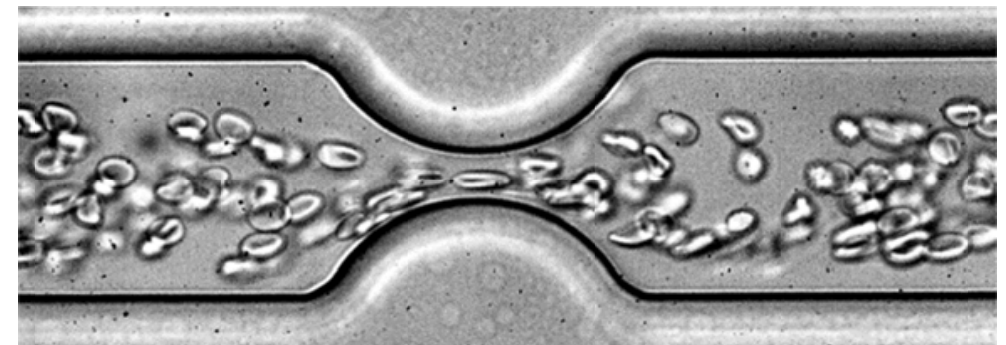

Figure 8. Blood flow visualization images of the deformation region of the CSD microfluidic device containing a smooth constriction microchannel with a width of $10 \mu \mathrm{m}$. 